\title{
ESTUDO COMPARATIVO DOS ANOS DE 2008 E 2018 DA PÓS-GRADUAÇÃO EM ENGENHARIA DE PRODUÇÃO
}

COMPARATIVE STUDY OF THE YEARS 2008 AND 2018 OF GRADUATE PROGRAMS IN PRODUCTION ENGINEERING Nathália de Miranda Barbosa ${ }^{1}$, Armando Araújo de Souza Júnior ${ }^{2}$ DOI: 10.37702/REE2236-0158.v40p473-482.2021

\section{RESUMO}

O conhecimento científico e tecnológico é desenvolvido a partir dos programas de pós-graduação. A inserção de novos mestres e doutores no meio acadêmico e empresarial é uma das formas de avaliar a efetividade e qualidade desses programas. Nesse sentido, este trabalho tem o objetivo de apresentar um estudo comparativo dos Programas de Pós-graduação em Engenharia de Produção no Brasil nos anos de 2008 e 2018, considerando a sua situação geográfica e a quantidade de docentes e discentes, e apresentar uma reflexão sobre o desenvolvimento da área de Engenharia de Produção. O estudo aponta que, apesar de os programas de Pós-graduação em Engenharia de Produção terem se desenvolvido, a sua distribuição geográfica está concentrada em algumas regiões do Brasil e houve uma redução na quantidade de dissertações defendidas, o que revela que é preciso intensificar os seus esforços para qualificar os programas e expandi-los em todas as regiões do país.

Palavras-chave: Pós-graduação; Engenharia de Produção; stricto sensu.

\begin{abstract}
Scientific and technological knowledge is developed based on postgraduate programs, in which it seeks to evaluate effectiveness and quality with the insertion of new masters and doctors in the academic and business environment. In this sense, this work aims to present a comparative study of graduate programs in production engineering in Brazil in the years 2008 and 2018, considering its geographical situation and the number of professors and students, to present a reflection on the development of the production engineering area. The study points out that, although postgraduate programs in production engineering have developed, their geographic distribution is concentrated in some regions of Brazil and there has been a reduction in the number of dissertations defended. In this way, postgraduate programs in production engineering need to intensify their efforts to qualify the programs and expand them in all regions of the country.
\end{abstract}

Keywords: postgraduate; Production Engineering; stricto sensu.

\footnotetext{
${ }^{1}$ Mestranda em Engenharia de Produção pela Universidade Federal do Amazonas, Av. General Rodrigo Octávio Jordão Ramos, 1200

- Coroado I, Manaus (AM), nathaliaviana3@hotmail.com

${ }^{2}$ Doutor em Administração pela Universidade Federal de Minas Gerais, Av. General Rodrigo Octávio Jordão Ramos, 1200 - Coroado

I, Manaus (AM), armandoaraujo@ufam.edu.br
} 


\section{INTRODUÇÃO}

A expansão dos programas de pósgraduação ao longo dos anos concorre para o incremento da produção científica nacional e para a melhoria da qualidade do serviço educacional fornecido pelas Instituições de Ensino Superior com a inserção de novos mestres, doutores e especialistas na academia brasileira (CIRANI; CAMPANARIO; SILVA, 2015).

É por meio das avaliações instituicionais, de face externa e interna, com o levantamento dos dados sobre a instituição, discentes e docentes que se pode avaliar a efetividade e qualidade do curso e das Instituições de Ensino Superior no tocante ao cumprimento de sua finalidade (NASCIMENTO et al., 2016).

A maior parte do conhecimento científico e tecnológico no país é desenvolvida no interior das universidades, especificamente por meio dos programas de pós-graduação (VOGEL; KOBASHI, 2015). Consequentemente, o crescimento dos indicadores de produção científica, titulados, cursos e programas da pósgraduação brasileira têm contribuído para a autonomia científica e tecnológica do país.

Com base nesse contexto, o objetivo deste trabalho é apresentar um estudo comparativo dos programas de pós-graduação em engenharia de produção no Brasil nos anos de 2008 e 2018, considerando a sua situação geográfica e a quantidade de docentes e discentes, apresentar uma reflexão sobre o desenvolvimento da área de engenharia de produção.

Para tanto, utilizou-se o levantamento de dados disponibilizados pela Capes e identificou-se as informações necessárias para atender ao objetivo proposto.

Logo, este trabalho está estruturado em seis seções: a) introdução; b) referencial teórico; c) resultados e discussões; d) conclusões finais; e) referências bibliográficas empregadas na pesquisa e, por fim, f) dados biográficos dos autores.

\section{Histórico da pós-graduação stricto sensu brasileira}

As origens da pós-graduação no Brasil datam de meados dos anos 1930, pois naquela época as primeiras universidades brasileiras conseguiram atrair alguns professores estrangeiros que vieram em missões acadêmicas apoiados por governos europeus ou, ainda, buscando terras brasileiras nos conturbados anos que antecederam a Segunda Guerra Mundial, e trazendo o primeiro modelo institucional para os estudos da pós-graduação (BALBACHEVSKY, 2005; SANTOS; AZEVEDO, 2009). Santos (2002) e Alves (2008) apontam que foi desta época a proposta do Estatuto das Universidades Brasileiras por Francisco Campos, então Ministro da Educação e Saúde Pública, o qual recomendou a implantação de uma pós-graduação nos moldes europeus.

A partir de 1940 foi formalmente utilizado o termo "pós-graduação" no Artigo 71 do Estatuto da Universidade do Brasil, e em 1950 se iniciaram acordos entre os Estados Unidos e o Brasil para implantação de convênios entre escolas e universidades americanas e brasileiras por meio do intercâmbio de estudantes, pesquisadores e professores (SANTOS, 2003; CURY, 2005).

Silva e Carvalho (2007) e Oliveira e Silva (2009) afirmam que surge, a partir de 1951, a atual CAPES, vinculada ao Ministério da Educação em um contexto de estímulo à formação técnico-científica, tendo como responsabilidade a formulação e a implementação da política nacional de pósgraduação. Nesse mesmo período, é criado o Conselho Nacional de Desenvolvimento Científico e Tecnológico (CNPQ), o qual era destinado ao fomento da pesquisa científica e tecnológica e à formação de recursos humanos para a pesquisa no país (MOREIRA, 2009).

Para Moritz, Moritz e Melo (2011) e Martins (2018), no início da década de sessenta foram criados os primeiros cursos na modalidade stricto sensu, destacando-se: mestrado e doutorado em Ciências Agrárias, na Universidade Federal de Viçosa e na Universidade Federal Rural do Rio de Janeiro; mestrado em Física e doutorado em Ciências 
Biológicas, na Universidade Federal do Rio de Janeiro; mestrado e doutorado em Matemática do Instituto de Matemática Pura e Aplicada, na Universidade de Brasília e Engenharia Aeronáutica no Instituto Tecnológico da Aeronáutica de São José dos Campos (MAer/ITA).

Na década de 1960 também houve a institucionalização da pós-graduação e da pesquisa, tendo a Universidade do Brasil desenvolvido uma iniciativa importante na área de Ciências Físicas e Biológicas, resultado de um convênio com a Fundação Ford, e outra na mesma universidade, na área de Engenharia, possibilitando assim a criação da Comissão Coordenadora dos Programas de PósGraduação em Engenharia - COPPE (SANTOS, 2003; FERREIRA, 2012).

A pós-graduação ganhou impulso, sobretudo, a partir da regulamentação por meio do Parecer $\mathrm{n}^{\circ}$ 977, de 3 de dezembro de 1965 , ficando conhecido como Parecer Sucupira, em homenagem ao professor Newton Sucupira por seu importante papel na implantação e organização da pós-graduação no Brasil (VELLOSO, 2014; NOBRE e FREITAS, 2017). Os autores afirmam ainda que o Parecer Sucupira estruturou a pós-graduação em dois níveis de formação, o stricto sensu e o lato sensu. Velloso (2004) e Balbachevsky (2005) destacam que, no ano de 1965, o Brasil possuía 38 cursos de pós-graduação, sendo 11 de doutorado e 27 de mestrado.

Germano (2005) e Saviani (2008) destacam a Lei 5.540/68, conhecida como Reforma Universitária de 1968, a respeito da qual se destacam a formação de quadros de alto nível, fomento da pesquisa e o desenvolvimento das ciências, sobretudo em termos de novas tecnologias e conhecimento aplicado para o crescimento econômico do país.

Com a percepção que a expansão da pósgraduação ocorria de forma relativamente espontânea, os contínuos governos militares foram instituindo medidas para garantir o seu desenvolvimento, com isso em 1970 foi criado o Programa Intensivo de Pós-graduação (Decreto 67.348/70), em 1973, foi criada uma equipe de trabalho com a finalidade de propor medidas iniciais para a definição da política de pós-graduação; e, em 1974, constituído o
Conselho Nacional de Pós-Graduação (Decreto 73.411/74), e formulou o primeiro Plano Nacional de Pós-Graduação (PNPG) para o período de 1975-1979 (FERREIRA, 1999; SANTOS; AZEVEDO, 2009).

O I PNG (1975-1979) tinha como principais destaques ampliar a capacitação dos docentes das instituições de ensino superior, distribuir regionalmente os cursos de pósgraduação, a valorização das ciências básicas e incentivar a produção científica (NUNES, FERRETO; BASTOS, 2010; CURVELO, 2018). Surge posteriormente o II PNPG (19821985), destacando a questão da qualidade e aperfeiçoamento da avaliação da pósgraduação (MELLO, 2002; SACARDO, 2007).

O III PNPG (1986-1989) enfatiza a importância da pós-graduação no desenvolvimento da pesquisa pela universidade, a incorporação da pós-graduação ao sistema nacional de ciência e tecnologia e a necessidade de procurar soluções aos problemas tecnológicos, sociais e econômicos (HOSTINS, 2006; OLIVEIRA; FONSECA, 2010). Segundo Morosini (2009) e Vogel (2015), o IV PNPG chegou a ser elaborado e passou pelas discussões nacionais, mas não se realizou como um plano oficial, no entanto, diversas recomendações que subsidiaram as discussões foram implantadas pela CAPES, tais como ampliação do sistema, diversificação do modelo de pós-graduação, alterações no processo de avaliação e inclusão internacional da pós-graduação.

Aprovado em janeiro de 2005, o V PNPG (2005-2010) teve como foco as políticas estratégicas do sistema educacional da pósgraduação no processo de desenvolvimento socioeconômico e cultural da sociedade brasileira (SANTOS; AZEVEDO, 2009; LIEVORE; PICININ; PILATTI, 2017).

Em 2010 foi aprovado o VI PNPG (20112020). O novo plano foi elaborado para fomentar a integração do ensino de pósgraduação com o setor empresarial e a sociedade, na expansão e correção de assimetrias regionais, criação de uma agenda nacional de pesquisa, aperfeiçoamento no modelo de avaliação da CAPES, incentivo à interdisciplinaridade e ações voltadas para elevar a qualidade da educação básica e outras 
modalidades de educação (ALVES; OLIVEIRA, 2014; NOBRE; FREITAS, 2017).

\section{Origem da Pós-Graduação Engenharia de Produção}

em

Em virtude da necessidade da instauração de empresas multinacionais no Brasil, bem como do desenvolvimento de indústrias nacionais, surgiu uma necessidade de gerenciamento e de aplicações de engenharia nessas indústrias, impulsionando o crescimento dos programas de engenharia de produção (OLIVEIRA, 2010; STURM et al., 2015).

Nesse contexto, Oliveira (2010) afirma que um dos principais aspectos que contribuem para o crescimento nos cursos de engenharia de produção é a evolução nos aspectos da produção. Ainda conforme o autor, a engenharia de produção é o curso que atende de forma mais completa as organizações, pois integra o conhecimento tecnológico ao sistêmico.

Bittencourt, Viali e Beltrame (2010) mencionam que, no ano de 1967, a PUC-RJ foi a pioneira ao criar o primeiro programa de mestrado em Engenharia de Produção no Brasil. Nesse mesmo ano foi criado o mestrado em Engenharia de Produção da Coppe/UFRJ e, na década de 1970, mais cinco programas de mestrado foram instituídos, entre eles, a da USP, UFF, UFPB, UFPE e UFSM.

Em 1972, a USP ofereceu o primeiro curso em nível de doutorado e somente a partir de 1979, o segundo curso de doutorado foi ofertado pela UFRJ. Em 1980 não se tem registros de criação de um programa ou curso de pós-graduação em engenharia de produção, no entanto, a partir de 1990 houve um grande crescimento no número de programas de mestrado (BITTENCOURT; VIALI; BELTRAME, 2010).

Segundo estudos de Bittencourt, Viali e Beltrame (2010) em um levantamento realizado em 2008 com base nas informações da CAPES, existiam 35 programas de engenharia de produção na área de engenharias III. Desse total, 17 programas ofertavam apenas o mestrado acadêmico, 12 programas ofertavam mestrado e doutorado, e 6 programas o mestrado profissional.

\section{RESULTADOS E DISCUSSÃO}

Santos e Azevedo (2009) mencionam que o sistema de pós-graduação no Brasil possui reconhecimento perante a comunidade científica, nacional e internacional. Esse fato deve-se à formação de políticas públicas para a pós-graduação implementada em termo de ações direcionadas para este setor que vem em contínua expansão nos últimos 40 anos, pois foi na década de 60 que a pós-graduação teve a sua institucionalização quando teve 38 cursos instalados no país.

Velloso (2004) acrescenta que a pósgraduação no país tem passado por notável expansão, além de ampliar sua abrangência quanto às áreas de conhecimento, foi instituída em meados dos anos 60 e dez anos depois, o número de cursos de pós-graduação já caminhava para um milhar.

O Gráfico 1 apresenta a quantidade de programas de pós-graduação em engenharia de produção no Brasil nos anos de 2008 e 2020. Em 2008 conforme Bittencourt, Viali e Beltrame (2010) existiam somente 35 programas de pós-graduação em engenharia de produção no Brasil, atualmente existem 54. Os programas de pós-graduação em engenharia de produção são ofertados por meio de cursos de Mestrado Acadêmico ou Profissional e de Doutorado Acadêmico ou Profissional.

Gráfico 1 - Quantidade de programas de pósgraduação em Engenharia de Produção nos anos de 2008 e 2020

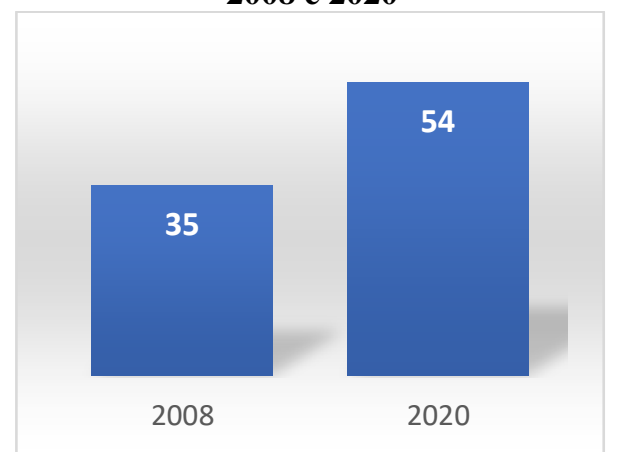

Fonte: elaborado pelos autores com base na Plataforma Sucupira/Capes, 2020.

Do total de programas de pós-graduação em engenharia de produção, 17 programas ofertavam apenas o mestrado acadêmico; 12 ofertavam mestrado e doutorado, e 6 programas o mestrado profissional (BITTENCOURT, 
VIALI e BELTRAME, 2010). A Engenharia de Produção, a qual pertence a área de Engenharias III, oferta seus cursos por meio de 15 Mestrados Acadêmicos, 15 Mestrados Profissionais, 23 Mestrados Acadêmicos e Doutorados Acadêmicos e 1 Mestrado Profissional e Doutorado Profissional, distribuídos nos níveis e modalidades conforme Tabela 1.

Verifica-se, por meio da Tabela 1, que houve uma diminuição na quantidade de mestrados acadêmicos em torno de $11 \%$, entretanto, teve um acréscimo de $150 \%$ referente aos mestrados profissionalizantes. Já os programas que oferecem mestrado e doutorado acadêmico tiveram um aumento em torno de $91 \%$ e os mestrados e doutorados profissionalizantes, que não contavam com nenhum programa, apresentaram um programa em 2020.

Tabela 1 - Cursos oferecidos pelo programa de pósgraduação em Engenharia de Produção nos anos de 2008 e 2020.

\begin{tabular}{|c|c|c|c|c|}
\hline ANO & ME & MP & ME/DO & MP/DP \\
\hline 2008 & 17 & 6 & 12 & 0 \\
\hline 2020 & 15 & 15 & 23 & 1 \\
\hline
\end{tabular}

Fonte: Elaborado pelos autores com base na Plataforma Sucupira /Capes, 2020.

Legenda: ME - Mestrado Acadêmico; DO - Doutorado Acadêmico; MP - Mestrado Profissional; ME/DO Mestrado Acadêmico/Doutorado Acadêmico; Mestrado Profissional/Doutorado Profissional.

O Gráfico 2 apresenta a distribuição dos programas do curso de engenharia de produção de acordo com as regiões do Brasil nos anos de 2008 e 2018. Bittencourt, Viali e Beltrame (2010) revelaram que os cursos de pósgraduação se concentravam nas regiões Sudeste e Sul, sendo que 25 municípios contavam com pelo menos um curso de pós-graduação em engenharia de produção e 9 municípios tinham, pelo menos, um curso de doutorado. Rio de Janeiro, São Paulo e São Carlos eram os únicos municípios com dois cursos de doutorado em funcionamento.

A região Centro-Oeste não contava com curso de pós-graduação na área, assim como quatro UFs da região Nordeste e o estado do Espírito Santo, no Sudeste. A região Norte, por sua vez, oferecia apenas um curso de mestrado profissional, oferecido na cidade de Manaus (BITTENCOURT; VIALI; BELTRAME, 2010).

No cenário atual, existem 12 municípios que ofertam pelo menos um programa de pósgraduação em engenharia de produção. As regiões que apresentam a maior quantidade de mestrados continuam sendo as regiões Sudeste e Sul, representadas pelos estados de Minas Gerais, Rio de Janeiro, São Paulo, Paraná, Santa Catarina e Rio Grande do Sul. Existem 8 municípios que ofertam programas de mestrado e doutorado acadêmicos, estando concentrados nas regiões Sudeste, Sul e Nordeste.

A região Nordeste, por meio do estado de Pernambuco, oferta o único mestrado e doutorado profissional. Já a região CentroOeste possui mestrado acadêmico e mestrado profissional no estado de Goiás e a região Norte continua sendo representada pelo estado do Amazonas, por meio de um mestrado profissional.

Gráfico 2 - Distribuição dos Programas de Engenharia de Produção nas regiões do Brasil nos anos de 2008 e 2018

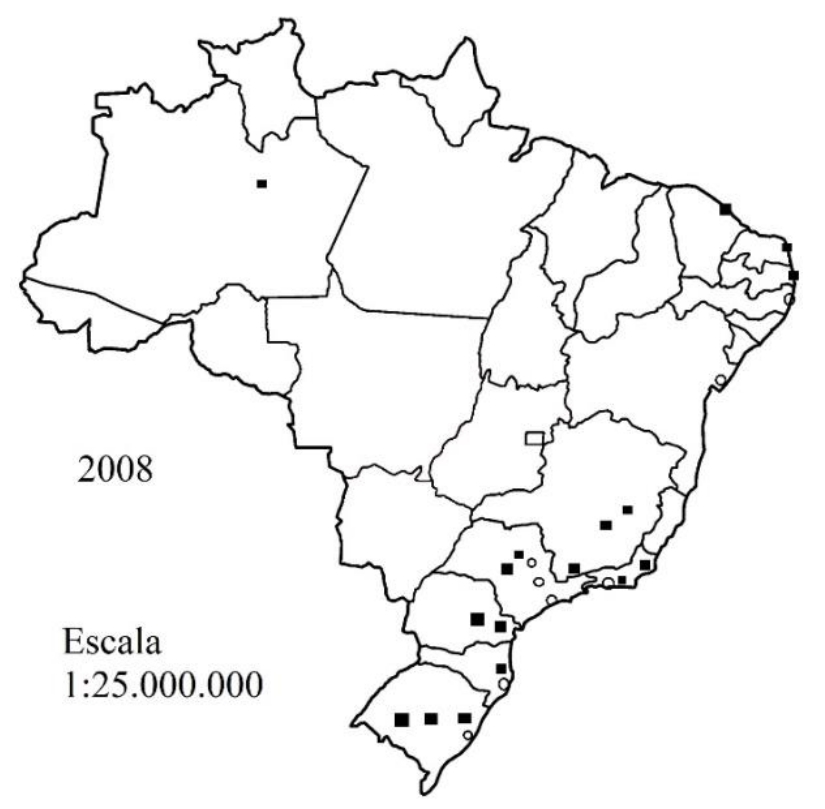




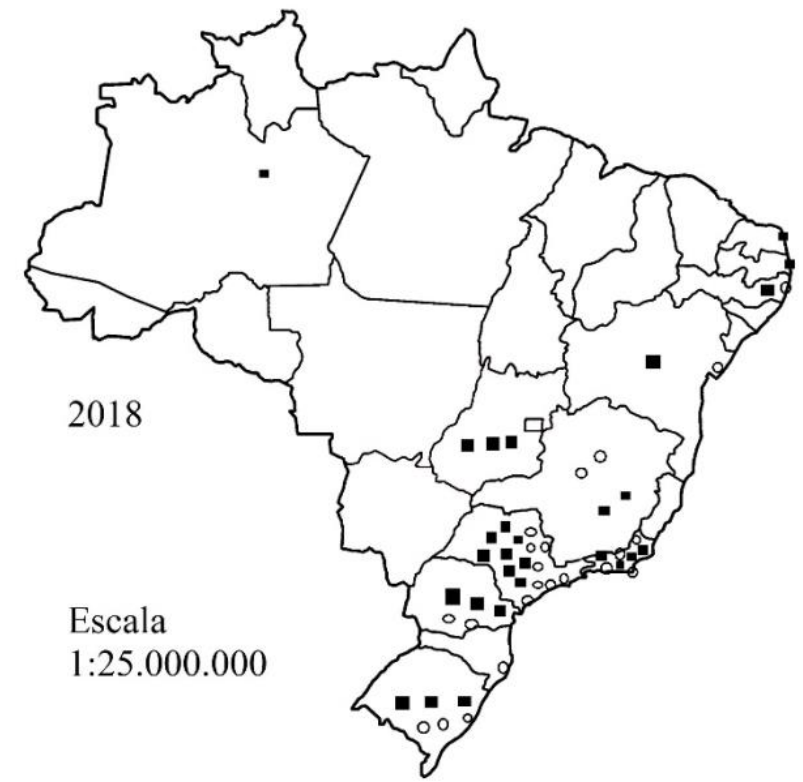

Fonte: Elaborado pelos autores com base na Plataforma Sucupira /Capes, 2020.

Legenda: $\square$ - Mestrado; O- Mestrado e Doutorado.

Em relação à qualidade dos cursos de mestrado e doutorado, os cinco cursos mais bem avaliados pela Capes, com conceito 5 ou superior, concentravam-se em universidades públicas, sendo o melhor deles exatamente um dos pioneiros, a UFRJ, tendo obtido a nota 6 ; porém, nenhum curso da área tinha atingido a nota 7 , considerada a nota máxima na escala de conceitos da Capes (BITTENCOURT; VIALI; BELTRAME, 2010).

A Tabela 2 apresenta o resultado da avaliação quadrienal de 2017, na qual doze cursos foram considerados bem avaliados pela Capes com conceito 5 ou superior, sendo a maioria concentrada ainda em universidades públicas. O programa de Mestrado Acadêmico e Doutorado Acadêmico da UFRJ foi o único a receber a nota 6 , mantendo a nota do ano de 2008. e foram avaliados com a nota 7 os programas de Mestrado Acadêmico e Doutorado Acadêmico da UFPE e UFRGS, que em 2008 não tinha sido obtida por nenhum curso.
Tabela 2 - Distribuição de notas dos programas de Engenharia de Produção

\begin{tabular}{|c|c|c|}
\hline INSTITUIÇÃO DE ENSINO & ME & ME/DO \\
\hline PUC-PR & 5 & 5 \\
\hline PUC-RIO & 5 & 5 \\
\hline USP-SC & 5 & 5 \\
\hline UNESP-BAURU & 5 & 5 \\
\hline UFBA & 5 & 5 \\
\hline UFMG & 5 & 5 \\
\hline UFSC & 5 & 5 \\
\hline UFSCAR & 5 & 5 \\
\hline UNIP & 5 & 5 \\
\hline UFRJ & - & 6 \\
\hline UFPE & - & 7 \\
\hline UFRGS & - & 7 \\
\hline
\end{tabular}

Fonte: Elaborado pelos autores com base na Plataforma Sucupira /Capes, 2020.

Legenda: ME - Mestrado Acadêmico; ME/DO Mestrado Acadêmico/Doutorado Acadêmico.

De acordo com a Capes (2008), no triênio de 2004 a 2006 foram defendidas 1.926 dissertações de mestrado e 269 teses de doutorado nesses 35 programas. Considerando a produção científica de qualis $\mathrm{A}$ ou $\mathrm{B}$ nesse período por meio do sistema qualis da Capes, em média 434 docentes por ano se envolveram no ensino da pós-graduação, tendo 249 artigos publicados em periódicos internacionais, 373 artigos em periódicos nacionais, 492 artigos em anais de eventos internacionais e $2180 \mathrm{em}$ eventos nacionais (BITTENCOURT; VIALI; BELTRAME, 2010).

O Gráfico 3 compara a quantidade de dissertações e teses defendidas durante o ano de 2008 e 2018. A Capes disponibilizou apenas até o ano de 2018, pois será atualizada após completar os quatros anos do ciclo (20172020), finalizando em 2021, ano da próxima avaliação quadrienal. Percebe-se que a quantidade de dissertações diminuiu em aproximadamente $4,8 \%$ no ano de 2018 , mas a quantidade de teses aumentou em aproximadamente $63,54 \%$. 
Gráfico 3 - Quantidade de dissertações e teses defendidas nos anos de 2008 e 2018

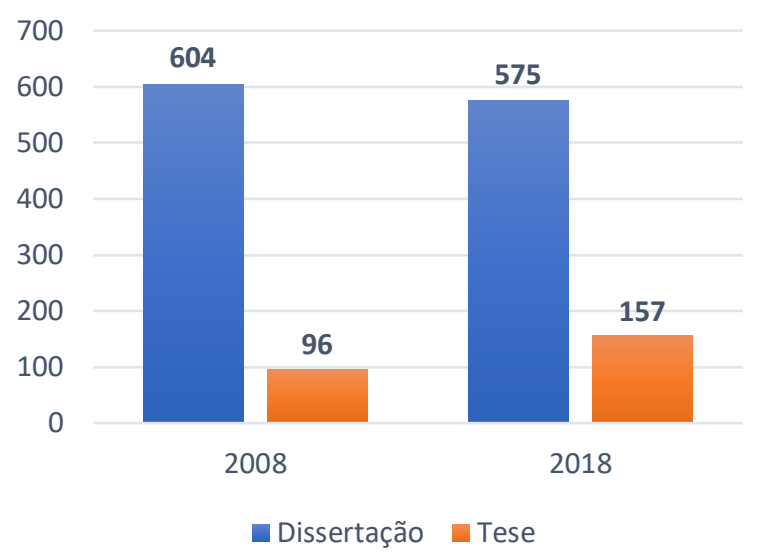

Fonte: elaborado pelos autores com base nos Dados Abertos/Capes, 2020.

O Gráfico 4 analisa a quantidade de docentes da pós-graduação em engenharia de produção, bem como a produção intelectual desses docentes, por meio da quantidade de artigos publicados em jornal ou revista, os artigos publicados em periódicos e os trabalhos em anais dos docentes nos anos de 2008 e 2018.

Verifica-se que a quantidade de docentes saltou em torno de $79,68 \%$ em relação ao ano de 2008; a publicação de artigos em jornal ou revista teve um acréscimo de aproximadamente $270 \%$. Já a publicação de artigos em periódicos teve um expressivo aumento de mais de $500 \%$, assim como os trabalhos em anais que aumentaram em aproximadamente $340,70 \%$.

\section{Gráfico 4 - Quantidade de docentes e produção intelectual nos anos de 2008 e 2018}

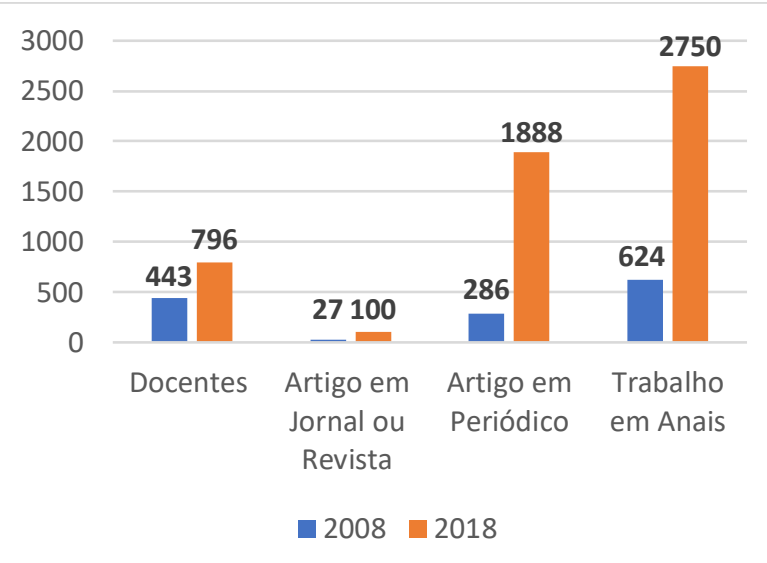

Fonte: Elaborado pelos autores com base nos Dados Abertos/Capes, 2020.
Os dados sobre os discentes da pósgraduação em engenharia de produção nos anos de 2008 e 2018 estão representados no Gráfico 5. A quantidade de discentes em 2018 em comparação com 2008 representa um aumento relativamente pequeno, aproximadamente $8,65 \%$. Desse total de discentes, $2,57 \%$ abandonaram o curso em 2008 e apenas $1,20 \%$ abandonaram em 2018.

Os alunos que se desligaram durante o curso em 2008 representaram 5,97\%, em 2018 esse número diminuiu, representando $3,51 \%$. Os alunos matriculados em 2008 eram de $68,61 \%$, já em 2018 os alunos matriculados eram de 73,24\%. Em relação aos alunos titulados, em 2008 eles representavam 22,08\%, e, em 2018, apenas 22,01\%.

Em 2008 havia a mudança de nível sem defesa; um total de 23 alunos fizeram tal opção, já em 2018 apenas um aluno solicitou mudança de nível sem defesa.

\section{Gráfico 5 - Quantidade de discentes e distribuição nos anos de 2008 e 2018}

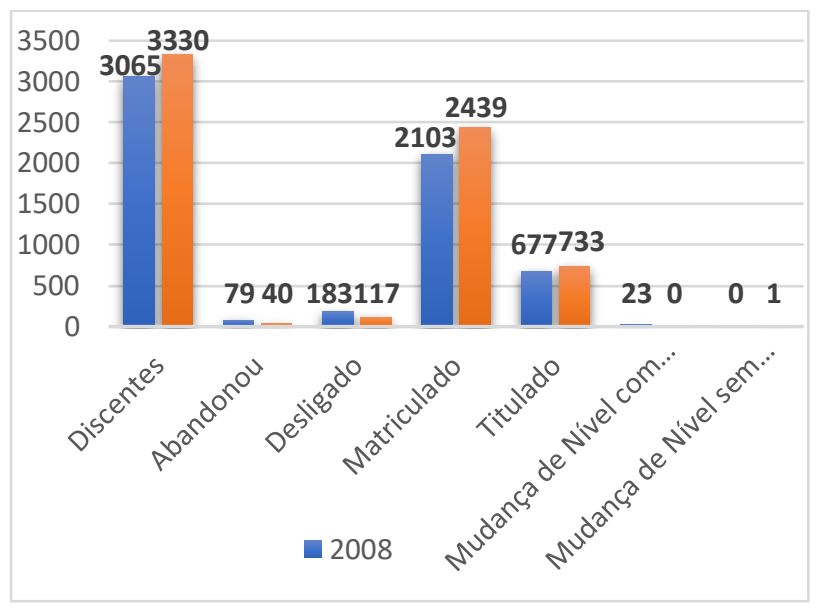

Fonte: Elaborado pelos autores com base nos Dados Abertos /Capes, 2020.

\section{CONSIDERAÇÕES FINAIS}

O estudo comparativo dos programas de pós-graduação em engenharia de produção permitiu analisar o crescimento da qualificação de mestres e doutores para o desenvolvimento da engenharia de produção no Brasil. Apontar as regiões que ofertam esses programas, bem como da avaliação dos cursos e da sua produção intelectual proporcionam uma análise crítica da área da engenharia de produção. 
Os resultados da pesquisa indicam que houve um aumento de $54,28 \%$ na quantidade de programas de 2008 para 2018. No entanto, as regiões que concentram a maior oferta de programa de pós-graduação em engenharia de produção continuam sendo as regiões Sudeste e Sul. Essa constatação, decorre da concentração dos maiores centros industriais e mercado consumidores do Brasil nessas regiões. $\mathrm{Na}$ região Sudeste, o único estado que ainda não possui nenhum programa é o Espírito Santo, apesar de apresentar destaque na agricultura, pecuária, mineração, petróleo e gás natural.

Apesar do aumento da oferta dos cursos de pós-graduação em engenharia de produção, os resultados do estudo indicam que houve uma redução das dissertações defendidas em aproximadamente $4,8 \%$. Por outro lado, a quantidade de teses defendidas obteve um crescimento de aproximadamente $63,54 \%$.

Ademais, verificou-se um aumento na quantidade de docentes de 2008 para 2018, assim como nas publicações de artigos em jornal ou revista, nos artigos em periódicos e os trabalhos em anais, resultantes da maior participação dos docentes em produção de conhecimento na área, principalmente devido às constantes mudanças no sistema de avaliação da CAPES, na qual reforçam a necessidade de dar maior atenção à qualidade de doutores e mestres que os programas estão formando de forma que contribua para a melhoria contínua.

A quantidade de discentes em 2018 teve um aumento relativamente pequeno, em comparação com 2008, verificou-se que poucos alunos abandonaram ou se desligaram do curso em 2018.

Em tempos de transformações tecnológicas intensas, a engenharia de produção possui um papel cada vez mais importante para a sociedade ao formar profissionais para atuarem nos mais diversos subsetores de atividade. Sob este prisma, os programas de pós-graduação em engenharia de produção precisam intensificar os seus esforços para qualificar os programas e expandi-los em todas as regiões do país.

\section{REFERÊNCIAS}

ALVES, V. M. Formação e trabalho de pesquisadores em educação: um estudo dos processos de institucionalização da pesquisa em IES "emergentes". Tese (Doutorado em Educação). Centro de Ciências da Educação, Universidade Federal de Santa Catarina, Florianópolis, 2008.

ALVES, M. F., OLIVEIRA, J. F. de. PósGraduação no Brasil: do Regime Militar aos dias atuais. Revista Brasileira de Política e Administração da Educação, Goiânia, v. 30, n. 2, p. 351-376, maio/ago., 2014.

BALBACHEVSKY, E. A pós-graduação no Brasil: novos desafios para uma política bemsucedida. In: Brock. C.; SCHWARTZMAN, S. Os desafios da educação no Brasil. Rio de Janeiro: Nova Fronteira, 2005.

BITTENCOURT, H. R.; VIALI, L.; BELTRAME, E. A engenharia de produção no Brasil: um panorama dos cursos de graduação e pósgraduação. Revista de Ensino de Engenharia, v. 29, n. 1, p. 11-19, 2010.

CIRANI, C. B. S., CAMPANARIO, M. D. A.; SILVA, H. H. M. S. A evolução do ensino da pós-graduação senso estrito no Brasil: análise exploratória e proposições para pesquisa. Revista da Avaliação da Educação Superior, v. 20, n. 1, p.163-187, 2015.

CURVELO, M. C. da S. O ensino no cenário da pós-graduação em Geografia no Brasil. 2018. 69f. Dissertação (Mestrado Profissional em Geografia). Centro de Ciências Humanas, Letras e Artes, Universidade Federal do Rio Grande do Norte, Natal, 2018.

CURY, C. R. J. Quadragésimo ano do parecer CFE $n^{\circ}$ 977/65. Revista Brasileira de Educação, ANPED, n. 30, p.7-20, set/dez - 2005.

FERREIRA, A. Desenvolvimento regional: limites e possibilidades institucionais: um estudo de caso da região do Vale do Paraíba RJ. Tese (Doutorado em Políticas Públicas, Estratégia e Desenvolvimento). Universidade Federal do Rio de Janeiro, 2012.

FERREIRA, R. A. A pesquisa científica nas ciências sociais: caracterização e 
procedimentos. Recife: Ed. Universitária da UFPE, 1999.

GERMANO. J. W. Estado militar e educação no Brasil (1964-1985). São Paulo: Cortez, 2005.

HOSTINS, R. C. L. Os Planos Nacionais de Pósgraduação (PNPG) e suas repercussões na Pósgraduação brasileira. PERSPECTIVA, Florianópolis, v. 24, n.1, p. 133-160, jan/jun. 2006.

LIEVORE, C.; PICININ, C. T.; PILATTI, L. A. As áreas do conhecimento na pós-graduação stricto sensu brasileira: crescimento longitudinal entre 1995 e 2014. Ensaio: Avaliação e Políticas Públicas em Educação, v. 25, n. 94, p. 207-237, 2017.

MARTINS, C. B. As origens da pós-graduação nacional (1960-1980). Revista Brasileira de Sociologia, v. 6, n. 13, 2018.

MELLO, K. Origem e Institucionalização da PósGraduação Strictu sensu Profissional: um Estudo de Casos. Teses e Dissertações. Recife: Universidade Federal de Pernambuco, 2002.

MIGUEL, P. A. C. Metodologia de pesquisa em engenharia de produção e gestão de operações. São Paulo: Elsevier-Campus, 2011.

MOREIRA, A. F. A cultura da performatividade e a avaliação da Pós-Graduação em Educação no Brasil. Educação em Revista, vol.25, n.3, p.23-42, dez., 2009.

MOROSINI, M. C. A pós-graduação no Brasil: Formação e desafios. Revista Argentina de Educación Superior, p.125-152, 2009.

MORTIZ, G. O.; MORTIZ, M. O; MELO, P. A. A pós-graduação brasileira: evolução e principias desafios no ambiente de cenários prospectivos. Anais... XI Colóquio Internacional sobre Gestão Universitária da América do Sul. Florianópolis, 2011.

NASCIMENTO, J. C. H. B. et al. Avaliação Institucional: Aplicação da Teoria da Resposta ao Item Para Avaliação Discente em Ciências Contábeis. Future Studies Research Journal: Trends and Strategies, v. 8, n. 2, p. 117-143, 2016.
NOBRE, N. L.; FREITAS, R. R. A evolução da pósgraduação no Brasil: histórico, políticas e avaliação. Brazilian Journal of Production Engineering, São Mateus, v.3, n.2, p.18-30, 2017.

NUNES, E. D.; FERRETO, L. E.; BARROS, N. F. DE. A pós-graduação em Saúde Coletiva no Brasil: trajetória. Ciênc. saúde coletiva, v. 15, n. 4, p.1923-1934, 2010.

OLIVEIRA, V. F. et al. Trajetória e estado da arte da formação em Engenharia, Arquitetura e Agronomia-volume I: Engenharias. Brasília: Instituto Nacional de Estudos e Pesquisas Educacionais Anísio Teixeira, Conselho Federal de Engenharia, Arquitetura e Agronomia, 2010.

OLIVEIRA, M. P.; SILVA, J. B. A trajetória da pós-graduação no Brasil e a ANPEGE: algumas questões. Revista da ANPEGE, v.5, p.79-82, 2009.

OLIVEIRA, J. F.; FONSECA, M. A pós-graduação brasileira e o seu sistema de avaliação. In: OLIVEIRA, J. F.; CATANI, A. M.; FERREIRA, N. S. C. (Org.). Pós-graduação e avaliação: impactos e perspectivas no Brasil e no cenário internacional. v. 1, Campinas: Mercado de Letras, 2010.

SACARDO, M. S. Reflexões acerca da pósgraduação brasileira: o impacto dessa política na área da Educação Física. Revista Brasileira de Ciências do Esporte, Campinas, v. 29, n. 1, p. 75-88, set., 2007.

SANTOS, C. M. Os primeiros passos da pósgraduação no Brasil: a questão da dependência. Ensaio: aval. pol. públ. educ. [online], vol. 10, n. 37, p.479-492, 2002.

SANTOS, C. M. Tradições e contradições da PósGraduação no Brasil. Educação e Sociedade. Campinas, v. 24, n. 83, p. 627- 641, 2003.

SANTOS, A. L. F.; AZEVEDO, J. M. L. A pósgraduação no Brasil, a pesquisa em educação e os estudos sobre a política: os contornos da constituição de um campo acadêmico. Revista Brasileira de Educação, v. 14, n. 42, p. 534605, set/dez, 2009. 
SAVIANI, D. O legado educacional do Regime Militar. Cadernos Cedes. Campinas, SP, vol.28, n.76, set./dez., 2008.

SILVA, M. O. da S.; CARVALHO, D. B. B. A Pósgraduação e a produção de conhecimento no serviço social brasileiro. Revista Brasileira de Pós-Graduação, Brasília, n. 4, v. 8, p. 192216, 2007.

STURM, C. H. et al. Mapeamento e análise de desempenho da graduação e da pós-graduação em Engenharia de Produção no Brasil. Gest. Prod., São Carlos, v. 22, n. 1, p. 149-163, mar. 2015.

VELLOSO, A. A pós-graduação no Brasil: legados e desafios. Almanaque Multidisciplinar de Pesquisa, v. 1, n. 1, p. 4-15, 2014.
VOGEL, M. J. M. Avaliação da pós-graduação brasileira: análise dos quesitos utilizados pela Capes e das críticas da comunidade acadêmica. 2015. Tese (Doutorado em Cultura e Informação) - Escola de Comunicações e Artes, Universidade de São Paulo, São Paulo, 2015.

VOGEL, M.; KOBASHI, N. Avaliação da pósgraduação no Brasil: seus critérios. Encontro Nacional de Pesquisa em Ciência da Informação, v. 16, p. 1-8, 2015.

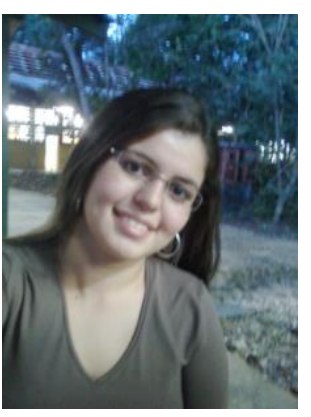

\section{DADOS BIOGRÁFICOS DOS AUTORES}

Nathália de Miranda Barbosa - Possui graduação em Ciências Contábeis pela Universidade Federal do Amazonas (2012). Especialização MBA em Gestão de Finanças, Controladoria e Auditoria pela Uninorte Laureate (2014). Mestranda de Engenharia de Produção pela Universidade Federal do Amazonas. Já atuou como assessora em órgãos públicos com ênfase em contratos e convênios. Atualmente exerce o cargo de Contadora na Universidade Federal do Amazonas.

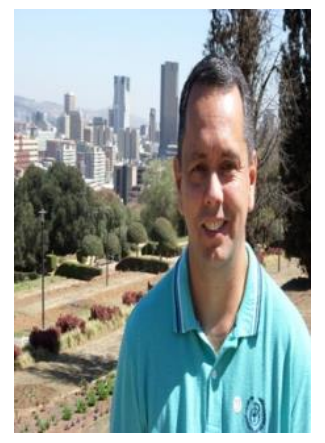

Armando Araújo de Souza Júnior - Possui graduação em Administração pelo CIESA (2001). Especialização MBA Executivo em Gestão da Produção pelo CIESA (2003), Especialização em Elaboração de Material Didático em Educação à Distância pela UFAM (2012), Especialização em Gestão de Hospitais Universitários no SUS pelo Instituo Sírio-Libanês de Ensino e Pesquisa (2014). Mestrado em Engenharia de Produção pela UFAM (2005). Doutor em Administração pela UFMG (2014). 